\title{
QUASISYMMETRIC MAPS AND STRING THEORY
}

\author{
By Osmo Pekonen
}

\begin{abstract}
We survey some recent research on the geometry of Lipman Bers' universal Teichmüller space $T(1)$, i.e., the space of quasisymmetric homeomorphisms of the circle modulo Möbius moves, and its applications in string theory.
\end{abstract}

\section{Geometric quantization}

Bosonic string theory $[9,13,26]$ is a proposal of unified field theory where the elementary particles called bosons are supposed to appear as 1-dimensional extended objects in the Planck scale $10^{-35} \mathrm{~m}$; hence, topologically they look like either $\mathbb{R}$ (open string) or $S^{1}$ (closed string). We shall work with closed strings. The string hypothesis introduces a new symmetry group into physics, the group Homeo $\left(S^{1}\right)$ of homeomorphisms of the circle, as this is the internal symmetry group of a closed string. Non-perturbatıve bosonic string theory would be based, ideally at least, on the group $\operatorname{Homeo}\left(S^{1}\right)$. We would like to geometrize this group, but as it seems to be intractable, in practice, we need to content ourselves with some subgroup.

There is a standard procedure in physics called geometric quantization [36] to pass from a classical system to a quantum system. In the classical system, the observables are functions $f$ in the phase space which is a smooth manifold $M^{2 n}$ endowed with a symplectic form $\omega$; in the corresponding quantum system the observables need to be converted into operators $T_{f}$ acting in some Hilbert space in such a way that Poisson brackets of functions are converted into Lie brackets of operators

$$
T_{\left\{f_{1}, f_{2}\right\}}=\left[T_{f_{1}}, T_{f_{2}}\right] \text {. }
$$

The standard way to achieve this is to produce a Hermitian line bundle $\mathcal{L}$ over $M$ with a Hermitian connection $\nabla$ whose curvature equals $\omega$. Then the sought-for operators will be given by

$$
T_{f}=-i \nabla_{X_{f}}+f
$$

where $X_{f}$ is the Hamiltonian vector field corresponding to the observable $f$ by the formula $X_{f}=-\omega^{-1}(d f,$.$) . The operators T_{f}$ act in the Hilbert space of square-integrable sections of $\mathcal{L}$ with respect to the canonical volume form $\frac{\omega^{n}}{n !}$ of $(M, \omega)$. In fact, up to this point, we have only achieved prequantization while the difficult Dirac problem concerning the irreducibility of the representation $f \mapsto T_{f}$ remains to be settled. This final step in the geometric quantization programme can often be achieved by introducing a

Received May 27, 1993. 
Kähler structure on the phase space and restricting to the holomorphic square-integrable sections.

Geometric quantization of string theory involves many unsolved problems which we shall discuss in due course later on. To begin with, we could try to produce a symplectic structure on Homeo $\left(S^{1}\right)$, or, more modestly, on some subgroup, e.g., the group $\operatorname{Diff}\left(S^{1}\right)$ of $C^{\infty}$ diffeomorphisms of the circle which should be more amenable to calculus procedures. The Lie algebra of the infinite-dimensional Lie group $\operatorname{Diff}\left(S^{1}\right)$ is the algebra $\operatorname{Vect}\left(S^{1}\right)$ of smooth vector fields on the circle. These have Fourier modes labeled by the integers $\mathbb{Z}$, so that $\operatorname{Vect}\left(S^{1}\right)$ formally behaves like an odd-dimensional space; hence, certainly Homeo $\left(S^{1}\right)$ or $\operatorname{Diff}\left(S^{1}\right)$ as such cannot carry a symplectic structure. Heuristically, an odd number of degrees of freedom ought to be removed before we can expect a symplectic phase space.

The simplest idea is to remove the Fourier zero mode by quotienting away the group of rotations $\operatorname{Rot}\left(S^{1}\right)$, or the circle itself. The resulting moduli space is denoted by

$$
N=\operatorname{Diff}\left(S^{1}\right) / \operatorname{Rot}\left(S^{1}\right) \text {. }
$$

Bowick and Rajeev [5] discovered that the space $N$ carries, indeed, the structure of an infinite-dimensional Kähler manifold. Implicitly, this phenomenon may be understood as an infinite-dimensional analogue of the finite-dimensional standard argument of Kirillov, Kostant, and Souriau which produces a symplectic structure in the coadjoint orbit space of a Lie group acting on the dual of its Lie algebra.

Moreover, there exists another obvious "even-dimensional" quotient space, namely

$$
M=\operatorname{Diff}\left(S^{1}\right) / \operatorname{Möb}\left(S^{1}\right) .
$$

Then $N$ is a holomorphic disc bundle over $M$. The Kirillov-Kostant-Souriau argument applies to $M$ as well, and, indeed, Witten [35] has proved that the dual of the Lie algebra of $\operatorname{Diff}(X)$ admits no other coadjoint actions by non-trivial subgroups of $\operatorname{Diff}(X)$. Hence, in principle, we can choose either $N$ or $M$ as the underlying phase space of our geometric quantization scheme. We shall see that $M$ is far more interesting.

\section{Universal Teichmüller space $T(1)$}

Between $\operatorname{Diff}\left(S^{1}\right)$ and Homeo $\left(S^{1}\right)$ there lies the group QS $\left(S^{1}\right)$ of quasisymmetric (qs) homeomorphisms. A homeomorphism $f$ is qs if it is $K$-qs for some $K$. The $K$-qs condition is most conveniently given in terms of the cross ratio

$$
\left(z_{1}, z_{2}, z_{3}, z_{4}\right)=\frac{z_{4}-z_{1}}{z_{4}-z_{2}} \frac{z_{3}-z_{2}}{z_{3}-z_{1}} \text {. }
$$

We need to require that

$$
\frac{1}{2 K} \leqq\left(f\left(z_{1}\right), f\left(z_{2}\right), f\left(z_{3}\right), f\left(z_{4}\right)\right) \leqq 1-\frac{1}{2 K}
$$

whenever $\left(z_{1}, z_{2}, z_{3}, z_{4}\right)=1 / 2$. Equivalently, quasisymmetric maps can be described as arising as boundary values of quasiconformal maps to be discussed later.

The fundamental sequence of subgroup inclusions

$$
\operatorname{Diff}\left(S^{1}\right)<\mathrm{QS}\left(S^{1}\right)<\operatorname{Homeo}\left(S^{1}\right)
$$

can be quotiented into

$$
M=\operatorname{Diff}\left(S^{1}\right) / \operatorname{Möb}\left(S^{1}\right) \subset \mathrm{QS}\left(S^{1}\right) / \operatorname{Möb}\left(S^{1}\right) \subset \operatorname{Homeo}\left(S^{1}\right) / \operatorname{Möb}\left(S^{1}\right) .
$$


Here we recognize a classical object; namely,

$$
T(1):=\mathrm{QS}\left(S^{1}\right) / \operatorname{Möb}\left(S^{1}\right)
$$

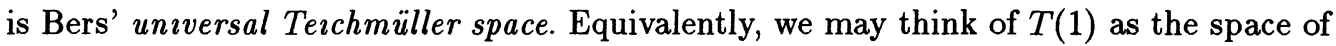
quasisymmetric homeomorphisms of the circle, say, which have three prescribed values. We stipulate the three points \pm 1 and $-i$ to be fixed. The infinite-dimensional space $T(1)$ is unıversal in the sense that it contains as subspaces all the other Teichmüller spaces whose definition we briefly recall next $[2,16,18,20,33]$.

Let $G$ be a Fuchsian group, i.e., a discrete subgroup of $\operatorname{Möb}(X)$. The Teachmüller space $T(G)$ is defined by

$$
T(G)=\left\{[f] \in T(1) \mid f \circ \gamma \circ f^{-1} \in \operatorname{Möb}(X) \text { for all } \gamma \in G\right\} .
$$

These spaces are partially ordered: $G<G^{\prime}$ clearly implies $T\left(G^{\prime}\right) \subset T(G)$; in particular, all the Teichmüller spaces $T(G)$ are contained in the universal one. (The " 1 " in the notation $T(1)$ refers to the trivial group.) Moreover, the inclusion $T(G) \subset T(1)$ turns out to be a holomorphic embedding.

Another approach to Teichmüller theory is from the point of view of uniformization. The Terchmüller space $T(\Sigma)$ of a Riemann surface $\Sigma$ is defined as the parametrization space of its complex structures up to isotopy. A complex structure may be given as a smooth section $J$ of the endomorphism bundle of the tangent bundle of the surface $\Sigma$ which is an anti-involution, i.e., $J^{2}=-$ id. Denote the space of all complex structures $J$ by $\mathcal{A}$. Of course, $\operatorname{Diff}(\Sigma)$ acts on $\mathcal{A}$ via the pull-back operation. In the case of a compact orientable surface of genus $>1$, the Teichmüller space $T(\Sigma)$ simply equals the moduli space $\mathcal{A} / \operatorname{Diff}_{0}(\Sigma)$ where $\operatorname{Diff}_{0}(\Sigma)$ is the identity component of $\operatorname{Diff}(\Sigma)$. This simple global analytic definition is powerfully exploited in the treatise [33].

In the above set-up, we can easily define a Kähler structure on $T(\Sigma)=\mathcal{A} / \operatorname{Diff}_{0}(\Sigma)$. By differentiating the relation $J^{2}=-$ id, we see that the tangent vectors $\dot{J}$ of $T(\Sigma)$ at $J$ anticommute with $J$ under composition of endomorphisms. Hence, the formula

$$
\omega\left(\dot{J}_{1}, \dot{J}_{2}\right)=\int_{\Sigma} \operatorname{tr}\left(J \circ \dot{J}_{1} \circ \dot{J}_{2}\right)
$$

defines a 2 -form on $\mathcal{A}$. The integration is with respect to the hyperbolic metric uniquely corresponding to $J$. This correspondence is natural with respect to the action of $\operatorname{Diff}(\Sigma)$; a fortiori, with respect to the action of the subgroup $\operatorname{Diff}_{0}(\Sigma)$, so it passes to the quotient. Moreover, it is straightforward to check that the resulting 2-form on the Teichmüller space $T(\Sigma)$ is non-degenerate and closed; hence, a Kähler form. This Kähler form (up to a constant multiple) is the classical Weil-Petersson Kähler 2-form.

If the surface $\Sigma$ is uniformized by a Fuchsian group $G, \Sigma=X / G$, then the two definitions coincide (Tukia [34]), $T(\Sigma)=T(G)$.

We shall briefly indicate how $T(\Sigma)$ and $T(G)$ are related to each other. For this purpose, we need to discuss the classical Beltramı equation. A homeomorphism $w$ : $D \rightarrow w(D)$ between domains in $\widehat{\mathbb{C}}$ is quasiconformal (qc) $[1,19]$ if and only if $w$ has locally integrable generalized derivatives satisfying almost everywhere on $D$ the Beltrami equation

$$
\bar{\partial} w(z)=\mu(z) \partial w(z)
$$

for some measurable complex function $\mu$ on $D$ called the Beltramı differentıal with

$$
\operatorname{ess} \sup _{z \in D}|\mu(z)|=\|\mu\|_{\infty}<1 \text {. }
$$


Denote the space of Beltrami differentials $L^{\infty}(D)_{1}$; it is the open unit ball in the complex Banach space $L^{\infty}(D)$ of essentially bounded functions in $D$. The existence and uniqueness, up to three prescribed values, of solutions for the equation (2.8) with an arbitrary Beltrami differential is guaranteed by a fundamental theorem due to Gauss, Morrey, Bojarski, and Ahlfors and Bers. We shall next discuss two pertinent classes of solutions corresponding to specially chosen Beltrami differentials $\mu \in L^{\infty}(\widehat{\mathbb{C}})_{1}$.

The real-analytic $w_{\mu}$-theory: By applying the fundamental existence and uniqueness theorem to the Beltrami differential which is $\mu$ on $\Delta$ and is extended to $\Delta^{\star}$ by reflection $\left(\tilde{\mu}(1 / \bar{z})=\overline{\mu(z)} z^{2} / \bar{z}^{2}\right.$ for $\left.z \in \Delta\right)$, one obtains the quasiconformal homeomorphism $w_{\mu}$ of $\mathbb{C}$ which is $\mu$-conformal in $\Delta$, fixes \pm 1 and $-i$, and keeps $\Delta$ and $\Delta^{\star}(=$ exterior of $\Delta$ ) both invariant.

The complex-analytic $w^{\mu}$-theory: By applying the existence and uniqueness theorem to the Beltrami differential which is $\mu$ on $\Delta$ and zero on $\Delta^{\star}$, one obtains the quasiconformal homeomorphism $w^{\mu}$ on $\mathbb{C}$, fixing $0,1, \infty$, which is $\mu$-conformal on $\Delta$ and conformal on $\Delta^{\star}$.

It is a fact that $w_{\mu}$ depends only real-analytically on $\mu$, whereas $w^{\mu}$ depends complexanalytically on $\mu$.

A Beltrami differential $\mu$ is G-equivariant, if it is compatible with the action of $G$ on $\Delta$; more precisely, this leads to the requirement

$$
\mu(\gamma z) \bar{\gamma}^{\prime}(z) / \gamma^{\prime}(z)=\mu(z)
$$

which should hold almost everywhere on $\Delta$ for every $\gamma \in G$. Let us denote the space of $G$-compatible Beltrami differentials $L^{\infty}(G)$. An alternative description of $T(G)$ can now be given as $T(G)=L^{\infty}(G) / \sim$ where $\mu \sim \nu$ if and only if $w_{\mu}=w_{\nu}$ on $\partial \Delta=S^{1}$, which happens if and only if $w^{\mu}=w^{\nu}$ on $\Delta^{*} \cup S^{1}$. Now, if $\mu$ is $G$-invariant, then $w_{\mu}$ conjugates $G$ to another Fuchsian group $G_{\mu}=w_{\mu} G w_{\mu}^{-1}$. The equivalence class of $\mu$ in $T(G)$ represents the Riemann surface $X_{\mu}=\Delta / G_{\mu}$.

In the reverse direction, one can use $w^{\mu}$ to conjugate $G$ to a quasi-Fuchsian group $G^{\mu}=w^{\mu} G\left(w^{\mu}\right)^{-1}$ so that $G^{\mu}$ acts discontinuously on the quasidisc $\Delta^{\mu}=w^{\mu}(\Delta)$ and its exterior $\Delta^{* \mu}=w^{\mu}\left(\Delta^{*}\right)$. Now, the Riemann surface $X_{\mu}$ is represented by $\Delta^{\mu} / G^{\mu}$ (whereas $\Delta^{* \mu} / G^{\mu}$ is the fixed Riemann surface $\Delta^{*} / G$, since $w^{\mu}$ is conformal on $\Delta^{*}$ ).

\section{Models' of $T(1)$}

We can think about $T(1)$ in several ways. The following three classical models of $T(1)$ are the best-known:

(a) the real-analytic model consisting of all Möbius-normalized quasisymmetric homeomorphisms of the unit circle $S^{1}$;

(a') the geometrıc model consisting of all Möbius-normalized quasıcırcles, i.e., all images of the standard circle under a global quasiconformal map that fixes the points \pm 1 and $-i$

(b) the complex-analytic model comprising all functions which fix $0,1, \infty$, which are univalent on the exterior of the unit disc $\Delta^{*}$ and which allow quasiconformal extension to the whole Riemann sphere.

As before, we may also define the universal Teichmüller space as a quotient of Beltrami differentials, $T(1)=L^{\infty}(\Delta)_{1} / \sim$ where $\mu \sim \nu$ if and only if $w_{\mu}=w_{\mu}$ on $\partial \Delta=S^{1}$, or equivalently, if and only if $w^{\mu}$ and $w^{\nu}$ coincide on $\Delta^{\star} \cup S^{1}$. 
We let $\Phi: L^{\infty}(\Delta)_{1} \longrightarrow T(1)$ denote the quotient projection. $T(1)$ inherits its canonical structure as a complex Banach manifold from the complex structure of $L^{\infty}(\Delta)_{1}$; indeed, $\Phi$ becomes a holomorphic submersion.

It is now clear that to $\mu \in L^{\infty}(\Delta)_{1}$ we can associate the quasisymmetric homeomorphism $f_{\mu}=\left.w_{\mu}\right|_{S^{1}}$ as representing the Teichmüller point $[\mu]$ in the real-analytic model (a) of $T(1)$. Indeed $T(1)_{(a)}$ is the homogeneous space $\mathrm{QS}\left(S^{1}\right) / \operatorname{Möb}\left(S^{1}\right)$ or the space of quasisymmetric homeomorphisms of $S^{1}$ fixing \pm 1 and $i$.

The geometric model ( $\left.\mathrm{a}^{\prime}\right)$ of $T(1)$ is merely a reformulation of (a): we can think of the points of $T(1)$ as the images of $S^{1}$ under $w_{\mu}$. There is a plethora of characterizations of the quasidiscs and their boundaries, the quasicircles [11]. Perhaps the most elegant is Ahlfors' condition which identifies quasicircles among those Jordan curves of the complex plane which pass through $\infty$ (this can be achieved by a Möbius transformation): Such a Jordan curve $C$ is a quasicircle if and only if there is a constant $M$ such that for any three distinct points $a, b, c$ on $C$ with $b$ between $a$ and $c$

$$
|b-a| \leqq M|c-a| \text {. }
$$

A generic quasidisc turns out to be a fractal object.

Alternatively, $[\mu]$ is represented by the univalent function $f^{\mu}=\left.w^{\mu}\right|_{\Delta^{\star}}$ on $\Delta^{\star}$, in the complex-analytic model (b) of $T(1)$. A more natural choice of the univalent function representing $[\mu]$ is to use a different normalization for the solution $w^{\mu}$ (since we have the freedom to post-compose by a Möbius transformation). In fact, let $W^{\mu}=M^{\mu} \circ w^{\mu}$ where $M^{\mu}$ is the unique Möbius transformation so that the univalent function (representing

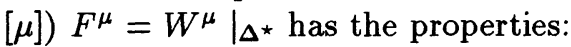

(i) $F^{\mu}$ has a simple pole of residue 1 at $\infty$;

(ii) $\left(F^{\mu}(z)-z\right) \rightarrow 0$ as $z \rightarrow \infty$.

Thus, the expansion of $F^{\mu}$ in $\Delta^{\star}$ is of the form:

$$
F^{\mu}(z)=z+\frac{b_{1}}{z}+\frac{b_{2}}{z^{2}}+\frac{b_{3}}{z^{3}}+\ldots
$$

Let us note that the original $(0,1, \infty$ fixing) normalization gives an expansion of the form:

$$
f^{\mu}(z)=z\left(a+\frac{\beta_{1}}{z}+\frac{\beta_{2}}{z^{2}}+\frac{\beta_{3}}{z^{3}}+\ldots\right)
$$

and the Möbius transformation $M^{\mu}$ must be $M^{\mu}(w)=w / a-\beta_{1} / a$. Since $\left(a, \beta_{1}, \beta_{2}, ..\right)$ depend holomorphically on $\mu$, we see that $\left(b_{1}, b_{2}, b_{3}, \ldots\right)$ also depend holomorphically on $\mu$. Thus, our complex-analytic version of $T(1)$ is:

$$
\begin{aligned}
& T(1)_{(b)}=\left\{\text { Univalent functions in } \Delta^{\star}\right. \text { with power series of the form (3.2), } \\
& \text { allowing quasiconformal extension to the whole plane }\} \text {. }
\end{aligned}
$$

In the general theory of univalent functions, the functions of the type (3.2) are known as the class $\Sigma$ [10]. It is not difficult to compute that the area of the corresponding quasidisc is

$$
A=\pi\left(1-\sum_{n=1}^{\infty} n\left|b_{n}\right|^{2}\right) .
$$

Of course, this is non-negative so that we deduce the classical Area Theorem [10, 18, 20] about the coefficients $b_{n}$ in the class $\Sigma$ :

$$
\sum_{n=1}^{\infty} n\left|b_{n}\right|^{2} \leqq 1
$$


We may think of the coefficients $b_{n}$ as coordinates on $T(1)$. A refinement of the Area Theorem shows that $b_{n}=\mathcal{O}\left(n^{-c}\right)$ with $c=0.509 \ldots$ [6], but the coefficients in the class $\Sigma$ still retain many mysteries. It is known that $\left|b_{1}\right| \leqq 1,\left|b_{2}\right| \leqq \frac{2}{3}$, and $\left|b_{3}\right| \leqq \frac{1}{2}+e^{-6}$. These bounds are sharp but there is no "Bieberbach conjecture" about the general sharp upper bound for $\left|b_{n}\right|$. Nonetheless, we can think of $T(1)$ as a space of certain sequences $\left(b_{1}, b_{2}, b_{3}, \ldots\right)$.

\section{The physicist's wish-list}

In this chapter, we explain why the universal property of $T(1)$ makes it an attractive object of study from the point of view of non-perturbative bosonic string theory whose precise geometric formulation, as we should stress, is unknown. First we shall review in simple non-technical terms the basic ideas of the prevailing perturbatıve bosonic string theory for the benefit of the reader who is not familiar with the physics literature.

The central issue in any quantum field theory is to evaluate the partition function $Z$ which gives the quantum-mechanical probability amplitudes of the system under study. Feynman introduced in 1948 a quantization scheme where $Z$ is computed as a path integral over the space of paths representing the possible worldlines of elementary particles. The possible spacetime trajectories of a propagating pointlike elementary particle are 1-dimensional paths, whereas a propagating bosonic string, or a 1-dimensional extended object, sweeps out 2-dimensional world-surfaces. A natural generalization of the Feynman path integral then is an integral over all possible world-sheets. As a first approximation, one can limit to deal with compact orientable surfaces which are topologically classified by the genus $\gamma=0,1,2, \ldots$ The emergence of a handle in the propagation pattern corresponds to the breaking apart of two strings; correspondingly, the annihilation of two strings closes a handle. In reality, one should also take into account the degenerate situations where, e.g., a handle shrinks to a node.

While the topological classification of compact orientable surfaces is easily understood, their geometrical diversity is more intricate. The possible geometries are given as the infinite-dimensional cone $\mathcal{M}$ of all Riemannian metrics $g$ of the underlying topological surface. This space, however, is physically redundant. The physically meaningful space in each genus $\gamma$ is the parametrization space of conformal structures, or, the Riemann moduli space $\mathcal{M}_{\gamma}$. It is well-known that $\mathcal{M}_{0}$ is a point, $\mathcal{M}_{1}=\mathbb{H} / \operatorname{PSL}(2 ; \mathbb{Z})$, while $\mathcal{M}_{\gamma}$ is an orbifold of formal dimension $6 \gamma-6$. The Riemann moduli space of a surface $\Sigma$ admits as its covering space the Terchmüller space $T(\Sigma)$, the parametrization space of conformal structures up to isotopy. Conformal structures are the relevant intrinsic geometries of the surface $\Sigma$, so one should develop an integration scheme over the moduli. Some explicit results are known, e.g., the volumes of some Teichmüller spaces in the Weil-Petersson metric (Penner [28]).

We think of $\Sigma$ propagating in a fixed background space that, as a first approximation, can be taken to be the flat Minkowski space of some dimension $D$. We also need to take into account the extrinsıc geometries of the string; in other words, the various ways in which a string may be embedded into the ambient space-time. An extrinsic metric is induced on the surface $M$ as a pull-back of the flat background metric via an embedding $s$. Thus we should also integrate over all embeddings $s: M \rightarrow \mathbb{R}^{D-1,1}$.

Consequently, we should be looking for the partition function in the form of a per- 
turbative series

$$
Z=\sum_{\gamma=0}^{\infty} \int_{\mathcal{M}_{\gamma}} e^{-S} .
$$

Here $S=S(g, s)$ is the Polyakov energy, i.e., the Dirichlet energy of an arbitrary embedding of the propagating string into the background spacetime. The integration in (4.1) is with respect to the so-called Polyakov measure over the moduli space $\mathcal{M}_{\gamma}$ of each genus $\gamma$ and also over the infinite-dimensional space of all embeddings $s$. Polyakov discovered in 1981 that this measure exhibits conformal anomaly cancellation in the critical spacetime dimension $D=26$.

Perturbative bosonic string theory suffers from several drawbacks. First of all, the summation over the genus in (4.1) is well-known to be infrared divergent [14]. Secondly, the need to prescribe the topology and geometry of the background spacetime is philosophically unsatisfactory. The spacetime should rather arise as an excitation. So far, merely the critical dimension $D=26$ arises as a constraint. Thirdly, the critical dimension is outlandish, be it lowered to the slightly more palatable $D=10$ in superstring theory [13] which incorporates fermions as well.

Perhaps these drawbacks indicate that we are just scratching the surface of some underlying intrinsic geometric principle that would imply more stringent conditions on the global properties of spacetime. The proper geometric environment of bosonic string theory should be some kind of "universal Riemann moduli space" which would comprise the moduli of surfaces with an arbitrary number of handles, cusps, boundary components, and nodes.

The only classically known universal moduli space in mathematics literature is Bers' universal Teichmüller space $T(1)$, although no viable notion of universal Riemann moduli space corresponds to it. Indeed, $T(1)$ is bound to be a highly relevant object, as it plays a role in both of the existing approaches to the quantization of bosonic strings:

(i) From the point of view of perturbatıve bosonic string theory, $T(1)$ contains as subspaces all the finite-dimensional Teichmüller spaces corresponding to various perturbative orders.

(ii) From the point of view of non-perturbatıve bosonic string theory, $T(1)$ is contained in the space Homeo $\left(S^{1}\right) / \operatorname{Mob}\left(S^{1}\right)$ which, in principle, should be the ultimate arena of the geometric quantization of bosonic string theory.

Perhaps the perturbative series (4.1) ought to be replaced by a single integral over the universal Teichmüller space

$$
Z=\int_{T(1)} e^{-S} .
$$

Some preliminary speculations about how the measure in $T(1)$ should look like in terms of the coefficients $b_{n}$ appear in [15], but no actual progress has been recorded.

The physicists' wish-list for mathematicians to achieve the geometric quantization of $T(1)$ includes (at least) the following items [29]:

(1) Unıversal geometry: $T(1)$ should be a Kähler manifold whose Kähler form $\omega$ pulls back to the Weil-Petersson form on each classical Teichmüller space $T(G)$.

(2) Universal topology: There should be an action by a "universal mapping class group" on $T(1)$ which pulls back to each $T(G)$. 
(3) Universal line bundle: There should exist a Hermitian line bundle $\mathcal{L}$ over $T(1)$ with a connection whose curvature equals $\omega$.

(4) Universal measure: $T(1)$ should carry a "Haar measure" with respect to which the classical locus, or the union of the images of the embeddings of all the $T(G)$, is dense and of measure zero in $T(1)$.

(5) Unıversal actıon princıple: There should exist a scalar-valued function $S$ ("universal Polyakov energy") whose gradient flow should have a superset of the classical locus as attracting fixed points. This function should coincide with a Kähler potential of the universal Weil-Petersson Kähler form.

We shall review the above-listed items emphasizing the established aspects of the theory. The current state of art seems to be that the item (1) is well-established for the quotient space $\operatorname{Diff}\left(S^{1}\right) / \operatorname{Möb}\left(S^{1}\right)$ while some evidence of its validity has been advanced in a suitable subspace of $T(1)$ (Nag and Sullivan [24]) and, from a different point of view, even in $\operatorname{Homeo}\left(S^{1}\right) / \operatorname{Möb}\left(S^{1}\right)$ (Penner [29]).

The solution to the item (2) has been claimed by Penner [29], but the discussion of his graph theoretic methods would bring us too far. Ratiu and Todorov [31] suggested that Quillen's determinant line bundle construction [30] applied to a family of CauchyRiemann operators parametrized by $M$ could solve the item (3). However, one sees with difficulty how Quillen's construction could be extended to $T(1)$. Possibly, ordinary calculus ought to be replaced by "quantum calculus" in the sense of Alain Connes' non-commutative geometry $[7,8]$.

The item (4) has been preliminarily discussed by Nag and Sullivan [24]. Some time ago, we suggested in [27] that the quasidisc area functional $A$ on $T(1)$ might serve as a heuristic candidate for the universal action principle required in item (5), because the quadratic expression in (3.4) can be interpreted as the Dirichlet energy of the harmonic extension in $\Delta$ defined by the boundary values of the univalent function $F^{\mu}$ in (3.2). However, we have not been able to compare the functional $A$ with the Polyakov energy of each genus.

\section{The almost complex structure of $T(1)$}

The Lie algebra of the Lie group $\operatorname{Diff}\left(S^{1}\right)$ is the Lie algebra $\operatorname{Vect}\left(S^{1}\right)$ of smooth vector fields on $S^{1}$. The complexification $\operatorname{Vect} \mathbb{C}_{\mathbb{C}}\left(S^{1}\right)$ of $\operatorname{Vect}\left(S^{1}\right)$ is generated by the Fourier modes

$$
L_{n}=e^{\imath n \vartheta} \frac{d}{d \vartheta}=i z^{n+1} \frac{d}{d z}, \quad n \in \mathbb{Z}
$$

with $z=e^{\imath \vartheta}$. To $\operatorname{Vect}_{\mathbb{C}}\left(S^{1}\right)$ there does not correspond any global Lie group, yet, Neretin has constructed a complex semigroup whose tangent cone is a convex cone in $\operatorname{Vect}_{\mathbb{C}}\left(S^{1}\right)$. The Lie bracket of $\operatorname{Vect}_{\mathbb{C}}\left(S^{1}\right)$ is given by the Witt law

$$
\left[L_{m}, L_{n}\right]=i(n-m) L_{m+n} .
$$

A tangent vector to the orbit space $M=\operatorname{Diff}\left(S^{1}\right) / \operatorname{Möb}\left(S^{1}\right)$ at its origin is a linear combination

$$
\vartheta=\sum_{m \neq 0, \pm 1} \vartheta_{m} L_{m}, \bar{\vartheta}_{m}=\vartheta_{-m}
$$

where $\vartheta=u(\vartheta) \frac{\partial}{\partial \vartheta}$ is the corresponding smooth real vector field on the circle and the $\vartheta_{m}$ are the Fourier coefficients of $u(\vartheta)$. The Lie algebra corresponding to the three 
missing modes $\vartheta_{-1}, \vartheta_{0}, \vartheta_{1}$, is $s l(2 ; \mathbb{R})$, of course. We may conjugate the series (5.3) by the conjugation operator $J$ to

$$
J \vartheta=\sum_{m \neq 0, \pm 1}-i \operatorname{sgn}(m) \vartheta_{m} L_{m}
$$

This is again a smooth vector field, but $J$ can be applied to a much wider class, the so-called Zygmund class which coincides with the tangent vectors to $T(1)$. Notice that $J^{2}=-$ id. Thus, as Kerckhoff (unpublished) first pointed out, the conjugation operation on Zygmund class vector fields on $S^{1}$ transmutes to the almost complex structure of $T(1)$.

\section{The Bers embedding of $T(1)$}

To provide a system of complex coordinates for $T(1)$ Bers embedded $T(1)$ as a holomorphically convex domain into the complex Banach space $B$ which consists of all functions $\phi(z)$, holomorphic in the lower half-plane, $\mathbb{L}$, with bounded Nehari norm defined by

$$
\|\phi\|=\operatorname{ess} \sup _{z \in L} 4\left|y^{2} \phi(z)\right| \text {. }
$$

In the complex-analytic model of $T(1)$, let $f^{\mu}$ represent a point of $T(1)$ and $w^{\mu}$ a quasiconformal extension of $f^{\mu}$ to the whole plane. Then the Bers embedding $T(1) \hookrightarrow B$ is defined by

$$
f^{\mu} \mapsto S\left(w^{\mu}\right)
$$

where $S$ is the Schwarzıan derivative

$$
S f=\left(\frac{f^{\prime \prime}}{f^{\prime}}\right)^{\prime}-\frac{1}{2}\left(\frac{f^{\prime \prime}}{f^{\prime}}\right)^{2}
$$

which annihilates Möbius moves. Direct computation gives the transformation rule

$$
S(f \circ g)=(S f \circ g)\left(g^{\prime}\right)^{2}+S g
$$

in particular, precomposition by $M \in \operatorname{Möb}\left(S^{1}\right)$ leaves the Schwarzian derivative invariant. The Bers embedding does not depend on the representative $f^{\mu}$, nor on the quasiconformal extension $w^{\mu}$, as one can show. Since an element $f^{\mu}$ is determined by the Beltrami differential $\mu$ up to a Möbius move, we may think of the Bers embedding as a function of $\mu$ as well. It then defines a holomorphic embedding of $T(1)$ into $B$ with respect to the complex structure of the Beltrami differentials.

\section{The Kähler structure of $T(1)$}

Nag and Verjovsky [25] proved that the natural inclusion $M \hookrightarrow T(1)$ is holomorphic. The proof amounts to showing that, if we write in (5.4)

$$
J \vartheta=u^{*}(\vartheta) \frac{\partial}{\partial \vartheta}
$$

then $u^{*}$ is essentially the Hilbert transform of $u$; this is not very difficult.

A more subtle result of Nag [25] endows a subspace of $T(1)$ with a Kähler structure and shows the inclusion $M \hookrightarrow T(1)$ to be a Kähler isometry onto its image. Recall that 
the existence of a symplectic form $\omega$ on $M$ is predicted by the theory of coadjoint orbits. To compute it explicitly, we impose the condition $d \omega=0$, or, equivalently, at the origin

$$
\omega\left(\left[L_{m}, L_{n}\right], L_{p}\right)+\omega\left(\left[L_{n}, L_{p}\right], L_{m}\right)+\omega\left(\left[L_{p}, L_{m}\right], L_{n}\right)=0 .
$$

Also, $\omega$ must vanish whenever one of its arguments is $L_{0}, L_{ \pm 1}$ since these vector fields give the zero tangent vector to $M$. The conditions (5.2) and (7.2) now lead to a system of difference equations whose only possible solution readily yields a homogenous Kähler form $\omega$ which is given at the origin by

$$
\omega\left(L_{m}, L_{n}\right)=\alpha\left(m^{3}-m\right) \delta_{m,-n}, \quad m, n \in \mathbb{Z} \backslash\{0, \pm 1\} .
$$

The constant $\alpha \in \mathbb{C} \backslash\{0\}$ is arbitrary.

Let $v=\sum_{m} v_{m} L_{m}$ and $w=\sum_{m} w_{m} L_{m}$ of the form (5.3) represent two tangent vectors to $M$ at the origin. Then the Kähler metric $g$, whose Kähler form $\omega$ was determined above, assigns the inner product

$$
g(v, w)=\alpha \operatorname{Re}\left(\sum_{m=2}^{\infty} v_{m} \bar{w}_{m}\left(m^{3}-m\right)\right) .
$$

According to standard results in harmonic analysis, the Fourier coefficients of a $C^{k+\epsilon}$ smooth function on $S^{1}$ decay at least as fast as $1 / n^{k+\epsilon}$. Hence, the infinite series in (7.4) converges absolutely whenever the vector fields $v$ and $w$ are $C^{3 / 2+\epsilon}$ smooth on $S^{1}$ for any $\epsilon>0$. Zygmund class functions are not necessarily smooth at all, so that the series (7.4) does not yield a well-defined inner product on all of $T(1)$. Claims have been made, though, that even Homeo $\left(S^{1}\right) / \operatorname{Möb}\left(S^{1}\right)$ carries a Kähler structure in some sense [29].

The Kähler structure $\omega$ is universal in the sense that it is closely related to the Weil-Petersson Kähler forms on each $T(G)$. However, the relationship is not by simple restriction of domains from the infinite-dimensional space $T(1)$ to the complex-analytic subspace $T(G)$, because $T(G)$ is transversal to the leaf $M$ of the foliation of $T(1)$ in the following sense: Let us use the geometric definition of $T(1)$ as the space of Möbiusnormalized quasidiscs. Bowen [3] proved the deep result that if $G$ uniformizes a compact Riemann surface, then every non-origin point of $T(G)$ corresponds to a quasidisc with fractal boundary. On the other hand, the quasidiscs corresponding to points of $M$ are the ones with $C^{\infty}$ boundaries. Nag [25] showed that every non-null tangent vector to $T(G)$ at the origin produces a vector field on $S^{1}$ that cannot be even $C^{3 / 2+\epsilon}$ smooth.

Nonetheless, the expression of the metric (7.4) is formally the same as that of the Weil-Petersson metric even when it diverges, and it can be regulated as explained by $\mathrm{Nag}$ in [25]. This procedure is not entirely satisfactory from the point of the physicists' wish-list, however.

\section{Curvature properties of $\operatorname{Diff}\left(S^{1}\right) / \operatorname{Möb}\left(S^{1}\right)$}

Conformal field theory [17] suggests that the natural value of the constant $\alpha$ is $\alpha=\frac{1}{12}$. This normalization is natural also when $\omega$ is viewed as the generator of the second Gelfand-Fuks cohomology $H^{2}\left(\operatorname{Vect}^{\infty}\left(S^{1}\right) ; \mathbb{C}\right.$ ) (Segal [32]). Besides being the unique symplectic form on $M$, the 2-cocycle $\omega$ can also be found as the unique central extension of the Lie algebra of vector fields on the circle. The centrally extended Lie algebra then is called the Virasoro algebra. It is customary to write $\alpha=\frac{c}{12}$ so that the Virasoro law 
reads as

$$
\left[L_{m}, L_{n}\right]=i(n-m) L_{m+n}+\frac{c}{12}\left(m^{3}-m\right) \delta_{m,-n}
$$

Remarkably, the spectrum of the values of the coupling constant $c$ admitting unitary representations of the Virasoro algebra (8.1) is continuous precisely for $c>1$ [12]. For $c<1$, the spectrum consists of the discrete series

$$
c=1-\frac{6}{(k+2)(k+3)}, \quad k=1,2,3, \ldots
$$

This phenomenon also shows that the value $c=1$, i.e., $\alpha=\frac{1}{12}$ is critical.

The Ricci curvature of the Kähler manifold $M$ has been computed by Bowick and Lahiri [4]. Such computations are surprising because an infinite-dimensional trace can be performed without any regularization. For the critical normalization $\alpha=\frac{1}{12}$ one obtains

$$
\text { Ricci }=-26 \times \omega .
$$

It is surprising to see the mysterious critical dimension $D=26$ of bosonic string theory emerge in this context! The critical occurrence of the number 26 in two seemingly disparate roles must be an instance of the subtle interplay of Feynman's quantization and geometric quantization of bosonic string theory rather than a mere numerical coincidence, yet this phenomenon has never been geometrically explained.

\section{Conclusion}

There seems to be emerging a fascinating interplay of Teichmüller theory and noncommutative geometry which may shed new light on crucial issues of non-perturbative string theory. We hope that our survey will be helpful for someone who is seeking his or her way through the maze of existing literature before tackling the forthcoming papers of Connes and Sullivan [8], Nag and Sullivan [24], and Penner (in preparation).

\section{REFERENCE}

[1] L.V. Ahlfors, Lectures on quasiconformal mappings, Van Nostrand, Princeton, 1966, Reprinted by Wadsworth \& Brooks/ Cole Advanced Books and Software, Monterey, California (1987).

[2] L. Bers, Finite-dimensional Teichmüller space and generalizations, Bull. Amer. Math. Soc. 5 (1981), 131-172.

[ 3 ] R. Bowen, Hausdorff dimension of quasicircles, Publ. Math. IHES 50 (1979), 259-273.

[ 4 ] M.J. Bowick, A. Lahiri, The Ricci curvature of Diff $S^{1} / S L(2, R)$, J. Math. Phys. 29 (1988), 1979-1981.

[5] M.J. Bowick, S.G. Rajeev, The holomorphic geometry of closed bosonic string theory and $\operatorname{Diff}\left(S^{1}\right) / S^{1}$, Nucl. Phys. B 293 (1987), 348-384.

[ 6 ] L. Carleson, P.W. Jones, On coefficient problems for univalent functions and conformal dimension, Duke Math. J. 66, (1992), 169-206.

[ 7 ] A. Connes, Géométrie non-commutative, Interéditions, Paris, 1990.

[ 8 ] A. Connes, D. Sullivan, Quantum calculus on $S^{1}$ and Teichmüller theory, IHES prerint (1993).

[9] E. D'Hoker, D.H. Phong, The geometry of string perturbation theory, Rev. Mod. Phys. 60 (1988), 917-1065.

[10] P.L. Duren, Univalent functions, Springer-Verlag, Berlin-Heidelberg-New York, 1983.

[11] F.W. Gehring, Characteristic properties of quasidisks, Sém. Math. Sup. 84, Presses Univ. Montréal, 1982. 
[12] P. Goddard, D. Olive, Kac-Moody and Virasoro algebras in relation to quantum physics, Int. J. of Mod. Phys. A 1 (1986), 303-414.

[13] M.B. Green, J.H. Schwarz, E. Witten, Superstring Theory I-II, Cambridge Univ. Press, Cambridge, 1987.

[14] D.J. Gross, V. Periwal, String perturbation theory diverges, Phys. Rev. Lett. 60 (1988), 21052108.

[15] D.K. Hong, S. Rajeev, Universal Teichmüller Space and Diff( $\left.S^{1}\right) / S^{1}$, Commun. Math. Phys. 135 (1991), 401-411.

[16] Y. Imayoshi, M. Taniguchi, An introduction to Teichmüller spaces, Springer-Verlag, Berlin Heidelberg - New York, 1992.

[17] M. Kaku, Strings, conformal fields, and topology. An introduction, Springer-Verlag, Berlin Heidelberg - New York, 1991.

[18] O. Lehto, Univalent functions and Teichmüller spaces, Springer-Verlag, Berlin - Heidelberg New York, 1987.

[19] O. Lehto, K.I. Virtanen, Quasiconformal mappings in the plane, Springer Verlag, Berlin - Heidelberg - New York, 1973.

[20] S. Nag, The complex analytic theory of Teichmüller spaces, Wiley-Interscience, New York, 1988.

[21] S. Nag, A period mapping in universal Teichmüller space, Bull. Amer. Math. Soc. 26 (1991), 280-287.

[22] S. Nag, Non-perturbative string theory and the diffeomorphism group of the circle, Topological and geometrical methods in field theory, Turku, Finland 1991, J. Mickelsson, O. Pekonen (eds.), World Scientific, Singapore, (1992), pp. 267-292.

[23] S. Nag, On the tangent space to the Teichmüller space, Annales Acad. Sci. Fennicae, 18 (2) (1993), 377-393.

[24] S. Nag, D. Sullivan, Teichmüller theory and the universal pewriod mapping via quantum calculus and the $H^{1 / 2}$ space on the circle, preprint (1993).

[25] S. Nag and A. Verjovsky, Diff( $\left.S^{1}\right)$ and the Teichmüller spaces, Parts I and II, Commun. Math. Phys. 130 (1990), 123-138, (Part I by S.N and A.V.; Part II by S.N.).

[26] P. Nelson, Lectures on strings and moduli space, Physics reports 147 (1987), 337-375.

[27] O. Pekonen, Quasidisks and string theory, Phys. Lett B 252 (1990), 555-557.

[28] R.C. Penner, Weil-Petersson volumes, J. Diff. Geom. 35 (1992), 559-608.

[29] R.C. Penner, Universal constructions in Teichmüller theory, Adv. in Math. 98 (1993), 143-215.

[30] D. Quillen, Determinants of Cauchy-Riemann operators over a Riemanninan surface, Funct. Anal. Appl. 19 (1) (1985), 31-34.

[31] T. Ratiu, A. Todorov, An infinite-dimensional point of view on Weil-Petersson metric, (unpublished manuscript).

[32] G.B. Segal, Unitary representations of some infinite-dimensional groups, Commun. Math. Phys. 80 (1981), 301-392.

[33] A.J. Tromba, Teichmüller theory in Riemannian geometry, Lectures in Mathematics ETH, Birkhäuser, 1992.

[34] P. Tukia, Quasiconformal extension of quasisymmetric mappings compatible with a Möbius group, Acta Math. 154 (1985), 153-193.

[35] E. Witten, Coadjoint orbits of the Virasoro group, Commun. Math. Phys. 114 (1988), 1-53.

[36] N.J.M. Woodhouse, Geometric quantization, Clarendon Press, Oxford, 1980.

DEPARTMENT OF MATHEMATICS

UNIVERSITY OF JYVÄSKYLÄ

P.O. BOX 35

FIN-40351 JYVÄSKYLÄ

FinLAND 